\title{
Criteria for starlike and convex functions of order $\alpha$
}

\author{
Neng $\mathrm{Xu}^{1 *}$ and Ding-Gong Yang ${ }^{2}$
}

\footnotetext{
"Correspondence: xun@cslg.edu.cn ${ }^{1}$ Department of Mathematics, Changshu Institute of Technology, Changshu, Jiangsu 215500, China Full list of author information is available at the end of the article
}

\begin{abstract}
Let $\mathcal{A}_{n}(n \in \mathbb{N})$ be the class of certain analytic functions $f(z)$ in the open unit disk $\mathbb{U}$ and $\mathcal{P}_{n}(\lambda)$ be the subclass of $\mathcal{A}_{n}$ consisting of $f(z)$ which satisfy $\left|f^{\prime \prime}(z)\right| \leqq \lambda(\lambda>0)$

in $\mathbb{U}$. Some properties for the class $\mathcal{P}_{n}(\lambda)$, which are the improvements of the previous results due to Ponnusamy and Singh (Complex Var. Theory Appl. 34:276-291, 1997), are discussed.
\end{abstract}

MSC: Primary 30C45

Keywords: starlike function; convex function; strongly starlike function; subordination

\section{Introduction}

Let $\mathcal{A}_{n}$ denote the class of functions of the form

$$
f(z)=z+\sum_{k=n+1}^{\infty} a_{k} z^{k} \quad(n \in \mathbb{N}=\{1,2,3, \ldots\})
$$

which are analytic in the open unit disk $\mathbb{U}=\{z \in \mathbb{C}:|z|<1\}$, and let $\mathcal{A}_{1}=\mathcal{A}$.

A function $f(z) \in \mathcal{A}$ is said to be in the class $\mathcal{S}^{*}(\alpha)$ in $\mathbb{U}$ if it satisfies

$$
\operatorname{Re} \frac{z f^{\prime}(z)}{f(z)}>\alpha \quad(z \in \mathbb{U})
$$

for some real $\alpha(\alpha<1)$. If $f(z) \in \mathcal{S}^{*}(\alpha)$ with $0 \leqq \alpha<1$, then $f(z)$ is said to be univalent and starlike of order $\alpha$ in $\mathbb{U}$. We denote $\mathcal{S}^{*}(0)=\mathcal{S}^{*}$. A function $f(z) \in \mathcal{A}$ is said to be in the class $\mathcal{C}(\alpha)$ if it satisfies

$$
\operatorname{Re}\left\{1+\frac{z f^{\prime \prime}(z)}{f^{\prime}(z)}\right\}>\alpha \quad(z \in \mathbb{U})
$$

for some real $\alpha(\alpha<1)$. If $f(z) \in \mathcal{C}(\alpha)$ with $0 \leqq \alpha<1$, then $f(z)$ is said to be univalent and convex of order $\alpha$ in $\mathbb{U}$. We write $\mathcal{C}(0)=\mathcal{C}$.

Let $f(z)$ and $g(z)$ be analytic in $\mathbb{U}$. Then we say that $f(z)$ is subordinate to $g(z)$ in $\mathbb{U}$, written $f(z) \prec g(z)$, if there exists a function $w(z)$ analytic in $\mathbb{U}$ which satisfies $w(0)=0,|w(z)|<1$ $(z \in \mathbb{U})$ and $f(z)=g(w(z))$ for $z \in \mathbb{U}$. If $g(z)$ is univalent in $\mathbb{U}$, then the subordination $f(z) \prec$ $g(z)$ is equivalent to $f(0)=g(0)$ and $f(\mathbb{U}) \subset g(\mathbb{U})(c f$. Duren [1]). 
A function $f(z) \in \mathcal{A}$ is said to be strongly starlike of order $\beta$ in $\mathbb{U}$ if it satisfies

$$
\frac{z f^{\prime}(z)}{f(z)} \prec\left(\frac{1+z}{1-z}\right)^{\beta}
$$

for some real $\beta(0<\beta \leqq 1)$. We denote this class by $\widetilde{\mathcal{S}}^{*}(\beta)$. Note that $\widetilde{\mathcal{S}}^{*}(1)=\mathcal{S}^{*}$.

Define

$$
\mathcal{P}_{n}(\lambda)=\left\{f(z) \in \mathcal{A}_{n}:\left|f^{\prime \prime}(z)\right| \leqq \lambda(\lambda>0 ; z \in \mathbb{U})\right\}
$$

Mocanu [2] considered the problem of finding $\lambda$ such that

$$
f(z) \in \mathcal{P}_{n}(\lambda) \text { implies } f(z) \in \mathcal{S}^{*} .
$$

Mocanu [2] has shown that:

Theorem A ([2]) If

$$
\lambda=\frac{n(n+1)}{2 n+1} \quad(n \in \mathbb{N})
$$

then $\mathcal{P}_{n}(\lambda) \subset \mathcal{S}^{*}$.

Ponnusamy and Singh [3] proved the following results.

Theorem B Let

$$
\begin{gathered}
\lambda_{n}=\frac{n(n+1)}{\sqrt{(n+1)^{2}+1}} \quad(n \in \mathbb{N}) . \\
\text { If } 0<\lambda \leqq \lambda_{n}, \text { then } \mathcal{P}_{n}(\lambda) \subset \mathcal{S}^{*}(\beta), \text { where } \\
\beta=\beta_{n}(\lambda)= \begin{cases}\frac{(n+1)(n-\lambda)}{n(n+1)+\lambda}, & \text { if } 0<\lambda \leqq \frac{n(n+1)}{n+2}, \\
\frac{n^{2}(n+1)^{2}-\left((n+1)^{2}+1\right) \lambda^{2}}{2\left(n^{2}(n+1)^{2}-\lambda^{2}\right)}, & \text { if } \frac{n(n+1)}{n+2} \leq \lambda \leq \lambda_{n} .\end{cases}
\end{gathered}
$$

Theorem $\mathrm{C}$ Let $0<\beta \leqq 1$ and

$$
\lambda_{n}^{\prime}=\frac{n(n+1) \sin \frac{\pi \beta}{2}}{\sqrt{1+(n+1)^{2}+2(n+1) \cos \frac{\pi \beta}{2}}} \quad(n \in \mathbb{N}) .
$$

If $0<\lambda \leqq \lambda_{n}^{\prime}$, then $\mathcal{P}_{n}(\lambda) \subset \widetilde{\mathcal{S}}^{*}(\beta)$.

It is easy to verify that Theorem B and Theorem C are better than Theorem A in two different ways.

In this paper we generalize and refine the above theorems. Furthermore we find $\lambda$ such that $f(z) \in \mathcal{P}_{n}(\lambda)$ implies $f(z) \in \mathcal{C}(\alpha)(\alpha<1)$. These results are sharp. 


\section{Main results}

To derive our first result, we need the following lemma due to Hallenbeck and Ruscheweyh [4].

Lemma Let $g(z)$ be analytic and convex univalent in $\mathbb{U}$ and $f(z)=g(0)+\sum_{k=n}^{\infty} a_{k} z^{k}(n \in \mathbb{N})$ be analytic in $\mathbb{U}$. If $f(z) \prec g(z)$, then

$$
z^{-c} \int_{0}^{z} t^{c-1} f(t) d t \prec \frac{1}{n} z^{-\frac{c}{n}} \int_{0}^{z} t^{\frac{c}{n}-1} g(t) d t
$$

where $\operatorname{Re}(c) \geqq 0$ and $c \neq 0$.

Now, we derive the following.

Theorem 1 Let $0<\lambda<n(n+1)(n \in \mathbb{N})$. Iff $(z) \in \mathcal{P}_{n}(\lambda)$, then

$$
\left|\frac{z f^{\prime}(z)}{f(z)}-1\right|<\frac{n \lambda}{n(n+1)-\lambda} \quad(z \in \mathbb{U}) .
$$

The bound $\frac{n \lambda}{n(n+1)-\lambda}$ in (2.1) is sharp.

Proof Let

$$
f(z)=z+\sum_{k=n+1}^{\infty} a_{k} z^{k} \in \mathcal{P}_{n}(\lambda) \quad \text { and } \quad 0<\lambda<n(n+1) \quad(n \in \mathbb{N})
$$

Then we have

$$
z f^{\prime \prime}(z)=n(n+1) a_{n+1} z^{n}+\cdots \prec \lambda z .
$$

Applying the lemma with $c=1$, it follows from (2.2) that

$$
\frac{1}{z} \int_{0}^{z} t f^{\prime \prime}(t) d t \prec \frac{\lambda}{n} z^{-\frac{1}{n}} \int_{0}^{z} t^{\frac{1}{n}} d t
$$

which yields

$$
f^{\prime}(z)-\frac{f(z)}{z} \prec \frac{\lambda z}{n+1},
$$

and hence

$$
\left|f^{\prime}(z)-\frac{f(z)}{z}\right|<\frac{\lambda}{n+1} \quad(z \in \mathbb{U}) .
$$

By (2.3) we can write

$$
f^{\prime}(z)-\frac{f(z)}{z}=\frac{\lambda w(z)}{n+1}
$$


where $w(z)$ is analytic in $\mathbb{U}$ with $w(0)=0$ and $|w(z)|<1(z \in \mathbb{U})$. Since

$$
f^{\prime}(z)-\frac{f(z)}{z}=n a_{n+1} z^{n}+\cdots,
$$

the function $w(z)$ in (2.5) satisfies $|w(z)| \leq|z|^{n}(z \in \mathbb{U})$ by the Schwarz lemma. Also (2.5) leads to

$$
\int_{0}^{z}\left(\frac{f^{\prime}(t)}{t}-\frac{f(t)}{t^{2}}\right) d t=\frac{\lambda}{n+1} \int_{0}^{z} \frac{w(t)}{t} d t
$$

In view of (2.6), we deduce that

$$
\begin{aligned}
\left|\frac{f(z)}{z}-1\right| & =\frac{\lambda}{n+1}\left|\int_{0}^{1} \frac{w(u z)}{u} d u\right| \leqq \frac{\lambda}{n+1} \int_{0}^{1} \frac{|w(u z)|}{u} d u \\
& \leqq \frac{\lambda|z|^{n}}{n+1} \int_{0}^{1} u^{n-1} d u<\frac{\lambda}{n(n+1)}
\end{aligned}
$$

and so

$$
\left|\frac{f(z)}{z}\right|>1-\frac{\lambda}{n(n+1)}>0 \quad(z \in \mathbb{U}) .
$$

Now, by using (2.4) and (2.7), we find that

$$
\begin{aligned}
\left|\frac{z f^{\prime}(z)}{f(z)}-1\right| & =\left|\frac{z}{f(z)}\right|\left|f^{\prime}(z)-\frac{f(z)}{z}\right| \\
& <\frac{\frac{\lambda}{n+1}}{1-\frac{\lambda}{n(n+1)}}=\frac{n \lambda}{n(n+1)-\lambda}
\end{aligned}
$$

for $z \in \mathbb{U}$, which shows (2.1).

For sharpness, we consider the function

$$
f(z)=z+\frac{\lambda}{n(n+1)} z^{n+1} \quad(z \in \mathbb{U})
$$

for $0<\lambda<n(n+1)$. Obviously $f(z) \in \mathcal{P}_{n}(\lambda)$. Furthermore we have

$$
\left|\frac{z f^{\prime}(z)}{f(z)}-1\right|=\left|\frac{\frac{\lambda}{n+1} z^{n}}{1+\frac{\lambda}{n(n+1)} z^{n}}\right| \rightarrow \frac{n \lambda}{n(n+1)-\lambda}
$$

as $z \rightarrow e^{\frac{\pi i}{n}}$. This completes the proof of Theorem 1 .

Next, we prove the following.

Theorem 2 Let $0<\lambda<n(n+1)(n \in \mathbb{N})$. Then

$$
\mathcal{P}_{n}(\lambda) \subset \mathcal{S}^{*}(\alpha),
$$


where

$$
\alpha=\alpha_{n}(\lambda)=\frac{(n+1)(n-\lambda)}{n(n+1)-\lambda} .
$$

The result is sharp, that is, the order $\alpha$ is best possible.

Proof If $f(z) \in \mathcal{P}_{n}(\lambda)$ and $0<\lambda<n(n+1)(n \in \mathbb{N})$, then an application of Theorem 1 yields

$$
1-\operatorname{Re} \frac{z f^{\prime}(z)}{f(z)}<\frac{n \lambda}{n(n+1)-\lambda} \quad(z \in \mathbb{U}) .
$$

Hence $f(z) \in \mathcal{S}^{*}(\alpha)$ where $\alpha=\alpha_{n}(\lambda)$ is given by (2.9).

For the function $f(z) \in \mathcal{P}_{n}(\lambda)$ defined by (2.8), we have

$$
\operatorname{Re} \frac{z f^{\prime}(z)}{f(z)}=\operatorname{Re}\left\{\frac{1+\frac{\lambda}{n} z^{n}}{1+\frac{\lambda}{n(n+1)} z^{n}}\right\} \rightarrow \frac{(n+1)(n-\lambda)}{n(n+1)-\lambda}=\alpha
$$

as $z \rightarrow e^{\frac{\pi i}{n}}$. Therefore the order $\alpha$ cannot be increased.

Remark 1 Let us compare Theorem 2 with Theorem B. Clearly

$$
n(n+1)>\lambda_{n} \quad \text { and } \quad \alpha_{n}(\lambda)>\beta_{n}(\lambda) \quad\left(0<\lambda \leqq \frac{n(n+1)}{n+2}\right) .
$$

Also, for $\frac{n(n+1)}{n+2} \leqq \lambda \leqq \lambda_{n}$, we have

$$
\begin{aligned}
\alpha_{n}(\lambda)-\beta_{n}(\lambda) & =\frac{(n+1)(n-\lambda)}{n(n+1)-\lambda}-\frac{n^{2}(n+1)^{2}-\left((n+1)^{2}+1\right) \lambda^{2}}{2\left(n^{2}(n+1)^{2}-\lambda^{2}\right)} \\
& =\frac{2(n+1)(n-\lambda)(n(n+1)+\lambda)-\left(n^{2}(n+1)^{2}-\left((n+1)^{2}+1\right) \lambda^{2}\right)}{2\left(n^{2}(n+1)^{2}-\lambda^{2}\right)} \\
& =\frac{n^{2}(n+1-\lambda)^{2}}{2\left(n^{2}(n+1)^{2}-\lambda^{2}\right)}>0 .
\end{aligned}
$$

Thus we conclude that Theorem 2 extends and improves Theorem B by Ponnusamy and Singh [3].

Taking

$$
\lambda=\frac{n(n+1)}{2 n+1} \quad \text { and } \quad \lambda=n,
$$

Theorem 2 reduces to the following.

Corollary 1 For $n \in \mathbb{N}$ we have

$$
\mathcal{P}_{n}\left(\frac{n(n+1)}{2 n+1}\right) \subset \mathcal{S}^{*}\left(\frac{1}{2}\right) \quad \text { and } \quad \mathcal{P}_{n}(n) \subset \mathcal{S}^{*}
$$

The results are sharp. 
Further, applying Theorem 1, we derive the following.

Theorem 3 Let $0<\beta \leqq 1$ and

$$
\tilde{\lambda}_{n}=\frac{n(n+1) \sin \frac{\pi \beta}{2}}{n+\sin \frac{\pi \beta}{2}} \quad(n \in \mathbb{N}) .
$$

If $0<\lambda \leqq \tilde{\lambda}_{n}$, then $\mathcal{P}_{n}(\lambda) \subset \widetilde{\mathcal{S}}^{*}(\beta)$ and the bound $\tilde{\lambda}_{n}$ cannot be increased

Proof Let

$$
0<\beta \leqq 1, \quad f(z) \in \mathcal{P}_{n}(\lambda) \text { and } \quad 0<\lambda \leqq \tilde{\lambda}_{n},
$$

where $\tilde{\lambda}_{n}$ is given by (2.11). Then $\tilde{\lambda}_{n} \leqq n$ and it follows from Theorem 1 that

$$
\left|\frac{z f^{\prime}(z)}{f(z)}-1\right|<\frac{n \tilde{\lambda}_{n}}{n(n+1)-\widetilde{\lambda}_{n}}=\sin \frac{\pi \beta}{2} \quad(z \in \mathbb{U}) .
$$

This implies that

$$
\left|\arg \frac{z f^{\prime}(z)}{f(z)}\right|<\frac{\pi \beta}{2} \quad(z \in \mathbb{U})
$$

Hence $f(z) \in \widetilde{\mathcal{S}}^{*}(\beta)$.

Furthermore, for the function $f \in \mathcal{P}_{n}(\lambda)$ defined by (2.8) and $\tilde{\lambda}_{n}<\lambda<n(n+1)$, we have

$$
\left|\frac{z f^{\prime}(z)}{f(z)}-1\right| \rightarrow \frac{n \lambda}{n(n+1)-\lambda}>\frac{n \tilde{\lambda}_{n}}{n(n+1)-\tilde{\lambda}_{n}}=\sin \frac{\pi \beta}{2}
$$

as $z \rightarrow e^{\frac{\pi i}{n}}$. This shows that $f \notin \widetilde{S}^{*}(\beta)$ and so the proof of Theorem 3 is completed.

Remark 2 Since $\tilde{\lambda}_{n}>\lambda_{n}^{\prime}(c f$. Theorem C) we see that Theorem 3 is better than Theorem C by Ponnusamy and Singh [3].

Finally we discuss the following.

Theorem 4 Let $0<\lambda<n(n \in \mathbb{N})$ and $0<\sigma \leqq 1$. Iff $(z) \in \mathcal{P}_{n}(\lambda)$, then

$$
\operatorname{Re}\left\{\sigma\left(1+\frac{z f^{\prime \prime}(z)}{f^{\prime}(z)}\right)+(1-\sigma) \frac{z f^{\prime}(z)}{f(z)}\right\}>\alpha \quad(z \in \mathbb{U}),
$$

where

$$
\alpha=\alpha_{n}(\sigma, \lambda)=\sigma \frac{n-(n+1) \lambda}{n-\lambda}+(1-\sigma) \frac{(n+1)(n-\lambda)}{n(n+1)-\lambda} .
$$

The result is sharp, that is, the bound $\alpha_{n}(\sigma, \lambda)$ cannot be increased.

Proof Let $f(z) \in \mathcal{P}_{n}(\lambda)$ and $0<\lambda<n$. Then, by (2.2) (used in the proof of Theorem 1) and the Schwarz lemma, we can write

$$
z f^{\prime \prime}(z)=\lambda w(z) \quad(z \in \mathbb{U}),
$$


where $w(z)$ is analytic in $\mathbb{U}$ and $|w(z)| \leq|z|^{n}(z \in \mathbb{U})$. Further, we deduce from (2.14) that

$$
f^{\prime}(z)-1=\int_{0}^{z} f^{\prime \prime}(t) d t=\lambda \int_{0}^{z} \frac{w(t)}{t} d t=\lambda \int_{0}^{1} \frac{w(u z)}{u} d u
$$

which leads to

$$
\begin{aligned}
\left|f^{\prime}(z)\right| & \geqq 1-\lambda \int_{0}^{1} \frac{|w(u z)|}{u} d u \\
& >1-\lambda|z|^{n} \int_{0}^{1} u^{n-1} d u \\
& >1-\frac{\lambda}{n}>0 \quad(z \in \mathbb{U}) .
\end{aligned}
$$

Also, by Theorem 2, we have

$$
\operatorname{Re} \frac{z f^{\prime}(z)}{f(z)}>\frac{(n+1)(n-\lambda)}{n(n+1)-\lambda} \quad(z \in \mathbb{U}) .
$$

Let us define the function $g(z)$ by

$$
g(z)=\sigma\left(1+\frac{z f^{\prime \prime}(z)}{f^{\prime}(z)}\right)+(1-\sigma) \frac{z f^{\prime}(z)}{f(z)}-\alpha
$$

where $0<\sigma \leqq 1$ and $\alpha$ is given by (2.13). Then $g(z)$ is analytic in $\mathbb{U}$ and

$$
\begin{aligned}
g(0) & =1-\alpha=1-\sigma \frac{n-(n+1) \lambda}{n-\lambda}-(1-\sigma) \frac{(n+1)(n-\lambda)}{n(n+1)-\lambda} \\
& =\sigma \frac{n \lambda}{n-\lambda}+(1-\sigma) \frac{n \lambda}{n(n+1)-\lambda}>0 .
\end{aligned}
$$

We claim that $\operatorname{Re} g(z)>0$ for $z \in \mathbb{U}$. Otherwise there exists a point $z_{0} \in \mathbb{U}$ such that

$$
\operatorname{Re} g(z)>0 \quad\left(|z|<\left|z_{0}\right|\right) \quad \text { and } \operatorname{Re} g\left(z_{0}\right)=0 .
$$

Thus, in view of (2.15)-(2.18) and (2.13), we find that

$$
\begin{aligned}
\sigma\left|z_{0} f^{\prime \prime}\left(z_{0}\right)\right| & =\left|f^{\prime}\left(z_{0}\right)\right|\left|g\left(z_{0}\right)+\alpha-\sigma-(1-\sigma) \frac{z_{0} f^{\prime}\left(z_{0}\right)}{f\left(z_{0}\right)}\right| \\
& \geqq\left|f^{\prime}\left(z_{0}\right)\right|\left|\operatorname{Re} g\left(z_{0}\right)+\alpha-\sigma-(1-\sigma) \operatorname{Re} \frac{z_{0} f^{\prime}\left(z_{0}\right)}{f\left(z_{0}\right)}\right| \\
& >\left(1-\frac{\lambda}{n}\right)\left(\sigma-\alpha+(1-\sigma) \frac{(n+1)(n-\lambda)}{n(n+1)-\lambda}\right) \\
& =\sigma \lambda>0 .
\end{aligned}
$$

This contradicts the expression (2.14). Hence, we say that $\operatorname{Re} g(z)>0(z \in \mathbb{U})$ and (2.12) is proved. 
For the function $f(z) \in \mathcal{P}_{n}(\lambda)(0<\lambda<n)$ defined by (2.8), we get

$$
\begin{aligned}
& \operatorname{Re}\left\{\sigma\left(1+\frac{z f^{\prime \prime}(z)}{f^{\prime}(z)}\right)+(1-\sigma) \frac{z f^{\prime}(z)}{f(z)}\right\} \\
& =\sigma\left(1+\operatorname{Re}\left\{\frac{\lambda z^{n}}{1+\frac{\lambda}{n} z^{n}}\right\}\right)+(1-\sigma) \operatorname{Re}\left\{\frac{1+\frac{\lambda}{n} z^{n}}{1+\frac{\lambda}{n(n+1)} z^{n}}\right\} \\
& \quad \rightarrow \sigma \frac{n-(n+1) \lambda}{n-\lambda}+(1-\sigma) \frac{(n+1)(n-\lambda)}{n(n+1)-\lambda}=\alpha
\end{aligned}
$$

as $z \rightarrow e^{\frac{\pi i}{n}}$. Therefore the bound $\alpha$ is best possible.

Making $\sigma=1$ in Theorem 4 , we have the following.

Corollary 2 Let $0<\lambda<n(n \in \mathbb{N})$. Then

$$
\mathcal{P}_{n}(\lambda) \subset \mathcal{C}\left(\frac{n-(n+1) \lambda}{n-\lambda}\right)
$$

The result is sharp. In particular, for $n \in \mathbb{N}$, we have

$$
\mathcal{P}_{n}\left(\frac{n}{2 n+1}\right) \subset \mathcal{C}\left(\frac{1}{2}\right), \quad \mathcal{P}_{n}\left(\frac{n}{n+1}\right) \subset \mathcal{C},
$$

and the results are sharp.

Taking $\sigma=\frac{1}{2}$ in Theorem 4 , we obtain the following.

Corollary 3 Let $0<\lambda<n(n \in \mathbb{N})$. Iff $(z) \in \mathcal{P}_{n}(\lambda)$, then

$$
\operatorname{Re}\left\{1+\frac{z f^{\prime \prime}(z)}{f^{\prime}(z)}+\frac{z f^{\prime}(z)}{f(z)}\right\}>\frac{n-(n+1) \lambda}{n-\lambda}+\frac{(n+1)(n-\lambda)}{n(n+1)-\lambda} \quad(z \in \mathbb{U}) .
$$

The result is sharp.

\section{Competing interests}

The authors declare that there is no conflict of interests regarding the publication of this article.

\section{Authors' contributions}

The main idea was proposed by NX and D-GY participated in the research. All authors read and approved the final manuscript.

\section{Author details}

'Department of Mathematics, Changshu Institute of Technology, Changshu, Jiangsu 215500, China. ${ }^{2}$ Department of Mathematics, Suzhou University, Suzhou, Jiangsu 215006, China.

\section{Acknowledgements}

This work was partially supported by the National Natural Science Foundation of China (Grant Nos. 11171045; 11471163).

The authors would like to express deep appreciation to Professor Shigeyoshi Owa for enlightening discussions and help. 
References

1. Duren, PL: Univalent Functions. Springer, New York (1983)

2. Mocanu, PT: Two simple sufficient conditions for starlikeness. Mathematica 34, 175-181 (1992)

3. Ponnusamy, S, Singh, V: Criteria for strongly starlike functions. Complex Var. Theory Appl. 34, 267-291 (1997)

4. Hallenbeck, DJ, Ruscheweyh, S: Subordination by convex functions. Proc. Am. Math. Soc. 52, 191-195 (1975)

Submit your manuscript to a SpringerOpen ${ }^{\circ}$ journal and benefit from:

- Convenient online submission

Rigorous peer review

- Immediate publication on acceptance

- Open access: articles freely available online

- High visibility within the field

- Retaining the copyright to your article 